Revista de Comunicación y Salud, 2020, Vol. 10, oㅡ 2, pp. 397-416

Editado por Cátedra de Comunicación y Salud

ISSN: 2173-1675

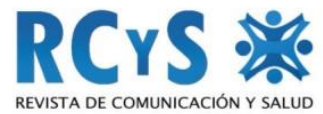

Enviado 12/08/2020

Aprobado 25/09/2020

\title{
LA FIGURA DEL PORTAVOZ EN LA COMUNICACIÓN DE UNA CRISIS SANITARIA: EL CASO DEL GOBIERNO ESPAÑOL DURANTE LA COVID-19
}

\section{The speaker figure in the communication of a health crisis: the case of the Spanish Government during the COVID-19}

\author{
José Miguel Rojo Martínez' \\ Universidad de Murcia. España. \\ josemiguel.rojo@um.es \\ Salvador Moreno Moreno \\ Universidad de Murcia. España. \\ salvador.moreno3@um.es \\ Alejandro Soler Contreras \\ Universidad de Murcia. España. \\ alejandro.solerc@um.es
}

\section{Resumen}

A lo largo del presente artículo se analiza, en perspectiva con la teoría disponible y tomando el estudio de caso como metodología fundamental, el ejercicio de la función de portavocía en el Gobierno de España durante la crisis sanitaria provocada por la COVID-19. Las clásicas variables sobre portavocía de crisis (perfil del elegido, número de portavoces, relación con los medios y capacidad decisoria o no de quien debe desarrollar esta labor) son evaluadas a la luz de la estrategia seguida por el Ejecutivo español, concluyendo que se ha optado por un sistema de portavocía múltiple, coordinada y mixta. Este formato ha planteado algunas disfuncionalidades y en él sobresalen como personajes destacados el doctor Fernando Simón, el ministro de Sanidad, Salvador Illa y el propio presidente del Gobierno, Pedro Sánchez. A los efectos de poder discutir las evidencias teóricas previas, se aportan indicadores sobre la comunicación de esta crisis y se recogen ciertos puntos conflictivos que podrían servir para una mejora futura de la transmisión de mensajes en este tipo de situaciones. Si hasta ahora ha quedado acreditado que no puede haber una buena gestión de crisis sin una correcta planificación de la comunicación, en el futuro, los nuevos escenarios sanitarios y ecológicos generarán contextos de amenaza masiva muy mediatizados para los que la correcta elección del portavoz de la crisis resultará determinante.

1 Autor para correspondencia: José Miguel Rojo Martínez, Universidad de Murcia, (josemiguel.rojo@um.es). 
La figura del portavoz en la comunicación de una crisis sanitaria: el caso del gobierno español durante la COVID-19

Palabras clave: Portavoz; Comunicación de crisis; Gestión de crisis; Comunicación de riesgo; España; Crisis sanitaria; COVID-19.

\begin{abstract}
Throughout this paper, the exercise of the speaker role in the Government of Spain during the health crisis caused by COVID-19 is analyzed in perspective with the available theory and taking the case study as fundamental methodology. The classic variables on crisis speaker (profile, number of speakers, relation to the media and decision-making capacity or not of who should carry out this work) are evaluated in the light of the strategy followed by the Spanish Executive, concluding that a coordinated and mixed multi-voice system has been chosen. This format has raised some dysfunctions and in it highlights include Doctor Fernando Simón, the Minister of Health, Salvador Illa and the President of the Government, Pedro Sánchez. In order to be able to discuss the previous theoretical evidence, indicators are provided on the communication of this crisis and some conflicting points are collected that could serve for a future improvement of messages transmission in this type of situation. If until now it has been proven that there cannot be good crisis management without proper communication planning, in the future, the new health and ecological scenarios will generate highly mediated massive threat contexts for which the correct choice of the crisis speaker will be decisive.
\end{abstract}

Keywords: Speaker; Crisis communication; Crisis management; Risk communication; Spain; Health crisis; COVID-19.

\title{
Cómo citar el artículo
}

Rojo Martínez, J., Moreno Moreno, S. y Soler Contreras, A. (2020). La figura del portavoz en la comunicación de una crisis sanitaria: el caso del gobierno español durante la COVID-19. Revista de Comunicación y Salud, 10 (2), 397-416. doi: https://doi.org/10.35669/rcys.2020.10(2).397-416

\section{INTRODUCCIÓN Y MARCO TEÓRICO}

Hace tiempo que las investigaciones académicas vienen poniendo de relieve la importancia de la dimensión comunicativa en un proceso de crisis. La orientación de la misma a la generación de certidumbres (Riorda, 2012) contribuye a que la percepción final que tiene la opinión pública sobre la gestión del Gobierno sea positiva y minimiza los daños sufridos por la institución. El interés político en este tipo de comunicación, por tanto, es evidente: una crisis puede hacer caer al más popular de los líderes.

Los "campos de pruebas" para la comunicación de crisis han abarcado los más diversos ámbitos, desde catástrofes naturales o colapsos financieros hasta huelgas de sectores económicos clave o escándalos políticos. En España, y dentro de la esfera sanitaria, no han sido pocas las crisis a las que se han tenido que enfrentar los cargos 
políticos. Desde el aceite de colza (1981) hasta la "enfermedad de las vacas locas" (2000) pasando por el brote de ébola (2014) o los recientes casos de listeriosis por carne mechada en mal estado (2019), las crisis sanitarias han supuesto algunas de las principales situaciones de estrés para Moncloa y las Comunidades Autónomas. Ninguno de estos casos, no obstante, reviste la importancia del que ahora nos ocupa. Por su magnitud inédita, la situación creada a partir de la pandemia de la COVID-19 se convierte en un momento crucial para las investigaciones en torno a la comunicación de crisis, poniendo a prueba todos los manuales sobre la disciplina.

Dentro de este marco, nuestro interés recae sobre un punto específico que, entre los diversos issues que comprende la comunicación de crisis, no ha recibido todavía un desarrollo teórico profundo: el papel de la portavocía. En la presente investigación, partimos de la bibliografía existente al respecto con el ánimo de complementarla y ampliarla mediante el análisis del caso de los portavoces del Gobierno de España durante esta crisis. Así pues, cotejamos con los postulados teóricos existentes la actuación de los diversos portavoces de la crisis mediante la construcción de indicadores y el análisis de la comunicación realizada y observamos diversas fallas adicionales que se dan durante el proceso y que pueden contribuir a la generación de nuevos debates a propósito de esta cuestión. En definitiva, de lo que se trata es de aplicar la teoría a un desempeño concreto para generar conocimiento científico ampliado desde una evidencia relevante.

\subsection{Comunicación de crisis y comunicación de riesgo como dos formas de comunicación política: una breve revisión.}

La comunicación política no se reduce al tradicional ámbito de las campañas electorales. Las principales necesidades de los políticos se concentran ahora fuera del periodo de votación y requieren de una creciente especialización en temas como la comunicación gubernamental o la comunicación de crisis, en el marco de una sociedad de riesgos crecientes (Beck, 1992).

Dentro de este contexto, el objetivo de la comunicación de crisis es "dotar de certidumbre y eliminar la conflictividad" (Riorda, 2011, p. 30). Para Olsson (2014, p. 9), la finalidad de este tipo de comunicación es "mantener la reputación y fomentar la resiliencia". La ausencia de planificación estratégica en la dimensión comunicativa de las crisis puede acabar "transmitiendo mensajes alarmistas a la población" y así "se contribuye a que las administraciones sean consideradas como sospechosas o de poca confianza" (Granda Revilla, 2015, p. 69). El daño reputacional que opera sobre líderes e instituciones como consecuencia de una gestión de crisis no estratégica y sin previsión ha impulsado una amplia producción científica sobre actuaciones recomendadas en estos casos. Podríamos resumir en dos grandes ítems las líneas maestras que repetidamente se señalan para conseguir una gestión comunicativa virtuosa de las crisis: a) "en comunicación de crisis la única opción es la verdad, aunque resulte dolorosa o perjudicial" y b) "es preferible pecar siempre por exceso de alarma que subestimar el daño o las consecuencias" (Riorda, 2012, pp. 39-40). 
Junto a la comunicación de crisis, la comunicación de riesgo aparece como "un proceso que conlleva la difusión de información relativa a los riesgos en que se encuentra una comunidad". La comunicación de riesgo es una acción eminentemente preventiva y educativa que persigue, por igual, evitar que aparezcan determinados perjuicios e informar con precisión a la ciudadanía para guiar su comportamiento hacia dinámicas seguras (Rosas Rodríguez y Barrios Puga, 2017, p. 183). Riorda (2011, pp. $21,33)$ distingue comunicación de riesgo de comunicación de crisis según se intente "clausurar un proceso" (en el caso de la segunda), o "modificar percepciones y generar un cambio en conductas" (para la primera). La Organización Panamericana de Salud [OPS] delimita el propósito de la comunicación de riesgo a que "toda persona expuesta a un riesgo sea capaz de tomar decisiones informadas para mitigar los efectos de la amenaza, como podría ser el brote de una enfermedad, y tomar las medidas y acciones de protección y prevención".

Tabla 1. Distinción entre comunicación de crisis y comunicación de riesgo.

\begin{tabular}{|c|c|c|c|}
\hline & Objetivos & Acciones & Ejemplo \\
\hline $\begin{array}{l}\text { Comunicación de } \\
\text { crisis }\end{array}$ & $\begin{array}{l}\text { Clausurar la } \\
\text { crisis ("salir"). } \\
\text { Evitar el daño } \\
\text { reputacional. } \\
\text { Aportar } \\
\text { certidumbre. } \\
\end{array}$ & $\begin{array}{l}\text { Constitución de un } \\
\text { comité de crisis, } \\
\text { comparecencias de } \\
\text { responsables } \\
\text { políticos. }\end{array}$ & $\begin{array}{l}\text { Rueda de prensa del } \\
\text { presidente del Gobierno de } \\
\text { España tras los atentados } \\
\text { del } 11-\mathrm{M} \text { (14:40 horas del } \\
11 \text { de marzo de } 2004 \text { en el } \\
\text { Palacio de La Moncloa). }\end{array}$ \\
\hline $\begin{array}{l}\text { Comunicación de } \\
\text { riesgo }\end{array}$ & $\begin{array}{l}\text { Modificar } \\
\text { conductas. } \\
\text { Informar. } \\
\text { Concienciar. }\end{array}$ & $\begin{array}{l}\text { Campañas públicas } \\
\text { de prevención. }\end{array}$ & $\begin{array}{l}\text { Publicidad institucional } \\
\text { para prevenir la infección } \\
\text { por VIH. }\end{array}$ \\
\hline
\end{tabular}

Fuente: elaboración propia a partir de Riorda (2011)

\subsection{El portavoz: rol decisivo para la comunicación de una crisis.}

Entre los "pasos estratégicos necesarios para controlar la crisis lo antes posible", March Cerdá (2011, p. 65) señala la obligación de "seleccionar un portavoz con formación en comunicación pública y que utilice de forma adecuada, clara y precisa los argumentarios preparados previamente por el equipo de gestión anticrisis". Profundizando en la importancia del portavoz dentro de la estrategia comunicativa para afrontar una crisis, este mismo autor identifica como uno de los errores "más habituales de una comunicación de riesgos en crisis de salud" que la organización no sea capaz de definir con claridad "a una persona que dé la cara, que sea portavoz" o que "quien hace de portavoz lo haga sin prepararse, sin pensar las consecuencias de sus intervenciones" (Cerdá, 2011, p. 62).

Muchos manuales de comunicación de crisis de distintas entidades ya comienzan a integrar y reconocer al portavoz como un elemento clave para asegurar el éxito en su gestión de este tipo de situaciones. Por ejemplo, el del Consejo de Seguridad Nuclear (CSN) español postula la necesidad de contar, como parte indispensable del proceso 
La figura del portavoz en la comunicación de una crisis sanitaria: el caso del gobierno español durante la COVID-19

comunicativo, con un portavoz fiable, único y con un entendimiento profundo de los aspectos técnicos de la crisis (CSN, 2012, p. 25).

De entre todas las crisis posibles (nucleares, naturales, de seguridad...), no podemos olvidar que las crisis relacionadas con el riesgo vital "han pasado de escenarios puramente sanitarios a entornos sociales y políticos donde el factor mediático se hace más relevante" (Moreno, 2008, p. 118). Justo por el impacto en el imaginario colectivo y en el devenir de las instituciones que tiene una crisis de salud, Crespo y Garrido (2020, p. 15) insisten en la idea de que "se requiere escoger cuidadosamente los portavoces de la crisis". Recuerdan, además, el consenso existente en los protocolos sobre la conveniencia de que "haya un portavoz único para evitar contradicciones en las comparecencias públicas", visión en la que coincide Medina (2017, p. 64), destacando el papel de los portavoces como "cara de la organización y quienes humanizan el mensaje". Además, si el portavoz es un "experto", se facilita para esta autora que "la comunicación durante una crisis sea mucho más eficiente y eficaz" (Medina, 2017, p. $65)$.

Paniagua Gallart (2012, pp. 12-13) argumenta que, si bien existe la idea inconsciente de que el portavoz de la crisis debe ser el máximo dirigente de la organización, es conveniente separar la portavocía habitual y la función de dirección de la portavocía de una crisis, evitando la hipoteca que supone para la imagen del dirigente el ser asociado para siempre a una situación negativa de crisis. Si hacemos un repaso a algunas de las crisis sanitarias de la España contemporánea (la encefalopatía espongiforme bovina, a principios de siglo, el brote de influenzavirus A en 2009 o el virus del ébola en 2014), en todas ellas han sido las ministras de Sanidad de la época, Celia Villalobos, Trinidad Jiménez y Ana Mato, respectivamente, quienes han actuado como portavoces y principales protagonistas de la comunicación de crisis.

Posiblemente como consecuencia del escaso éxito de las estrategias de gestión de crisis lideradas comunicativamente por políticos, como sucedió con las ministras señaladas, la literatura actual coincide en apostar por un portavoz técnico por encima del portavoz político. En el reciente libro Comunicación política en tiempos de coronavirus, el consultor Xavier Peytibi (2020, p. 13) entiende como una necesidad ineludible para afrontar una crisis "tener un buen portavoz" y que el mismo "sea experto, es decir, epidemiólogo en este caso, y que informe puntualmente cada día. Tiene mucha más credibilidad que un portavoz político. Se trata de que sea notario y no protagonista". Sobre la dicotomía entre "portavoz experto" y "portavoz político" reflexiona expresamente Verónica Fumanal (2020, pp. 23-27) en el mismo libro y lo hace sobre la base de tres variables decisivas: "credibilidad, atractivo y poder". Fumanal postula una mayor adecuación del portavoz técnico al criterio de credibilidad, mientras que el portavoz político cuenta inherentemente con un mayor atractivo y poder. Subyace a esta dicotomía la elección de los atributos del liderazgo preferentes, que se verán influidos por las necesidades del contexto en función de si se busca construir una legitimación vía racionalidad técnico-científica o por el componente más decisorio e ideológico-discursivo.

Revista de Comunicación y Salud, 2020, Vol. 10, nº 2, pp. 397-416 
La figura del portavoz en la comunicación de una crisis sanitaria: el caso del gobierno español durante la COVID-19

En cualquier caso, el portavoz de una crisis, técnico o político, va a ser el rostro socialmente reconocido de las decisiones adoptadas y va a tener el delicado encargo de transmitir todos los mensajes que la institución construya al respecto. Saló Lloveras (2008, p. 23) puntualiza que "el portavoz no sólo es responsable de los mensajes que se transmiten, sino que también debe estar involucrado en su elaboración para hacerlos suyos". No puede haber una gestión de crisis exitosa sin una buena comunicación, pero tampoco puede haber una buena comunicación de crisis sin un correcto ejercicio de la función de portavocía, porque "la actuación del portavoz puede condicionar la percepción en los públicos de la gestión de la comunicación de una crisis" (Crespo et al., 2017, p. 126), de ahí que cada vez más sea necesario centrar la atención de los estudios en esta figura.

\section{OBJETIVOS}

La presente investigación se articula a partir del objetivo general de analizar la función de portavocía de crisis en el caso del Gobierno de España durante la pandemia provocada por la COVID-19 considerando los aspectos teóricos ya presentes en los estudios sobre la materia. Para lograr lo anterior será necesario alcanzar y desarrollar una serie de objetivos específicos:

a) Identificar el modelo de portavocía elegido por el Gobierno español atendiendo a variables como el perfil de los portavoces, el número de portavoces, la capacidad decisional del portavoz o la relación con los medios.

b) Elaborar una serie de indicadores que nos permitan cuantificar las cuestiones más relevantes de una portavocía de crisis.

c) Conocer las estrategias comunicativas de transmisión de mensajes elaboradas por el Gobierno de España y evaluar su implementación por parte de los portavoces elegidos concluyendo aquellos aspectos susceptibles de mejora.

La consecución de nuestro catálogo de objetivos nos permitirá responder a las dos preguntas de investigación que se plantean:

I. P1. ¿Qué modelo de portavocía de crisis ha puesto en marcha el Gobierno español?

II. P2. ¿Se ha ajustado el Gobierno español a los parámetros clásicos y recomendaciones teóricas sobre la función de portavoz en una crisis?

\section{METODOLOGÍA}

La metodología que seguiremos es el estudio de caso único, lo que nos permitirá un análisis intensivo y profundo de la unidad seleccionada (el Gobierno español). Concretamente, y siguiendo a Lijphart (1971), nuestro estudio de caso es de tipo "desviado". Se trata de una investigación descriptiva de un caso crucial que pone a prueba las recomendaciones teóricas más frecuentes por su carácter extraordinario respecto al resto de casos-tipo que suelen tratarse en dicho ámbito de investigación. En este sentido, también se podría considerar nuestro ejercicio como un estudio de caso "debilitador de la teoría". Al elegir como metodología de investigación el estudio de caso no procede la formulación de hipótesis, no hay posibilidad de contraste, pero sí que 
La figura del portavoz en la comunicación de una crisis sanitaria: el caso del gobierno español durante la COVID-19

resulta pertinente plantear una proposición teórica, que es la siguiente: el Gobierno español se ha alejado del modelo teórico recomendado de portavocía de crisis. Y esto ha sucedido por la naturaleza excepcional de esta crisis, diferente de cualquier situación antes estudiada y que por sus efectos devastadores y su impacto transversal constituye en sí misma una impugnación a la acumulación de evidencias académicas previas.

Junto a una descripción general del método seguido, las técnicas de investigación aplicadas con sus correspondientes fuentes de información han sido: revisión teórica en profundidad de la bibliografía disponible; análisis diario por parte del equipo de investigación de las ruedas de prensa durante un periodo temporal seleccionado (del 12 de marzo al 26 de abril, el momento álgido de la crisis); sistematización del contenido de los vídeos disponibles en la plataforma YouTube o en los perfiles de redes de Moncloa que impliquen transmisión de mensajes; seguimiento de noticias de medios sobre las acciones de portavocía en la crisis; construcción de indicadores a partir del análisis realizado y tratando datos facilitados por la Secretaría de Estado de Comunicación en diferentes informes.

\section{DISCUSIÓN. RESULTADOS DE LA INVESTIGACIÓN}

\subsection{La elección del portavoz}

Una de las decisiones más importantes para gestionar correctamente la comunicación de crisis es la elección del portavoz. Esta decisión pasa, en primer lugar, por preguntarse si lo más adecuado es que la labor sea realizada por una sola persona o, por el contrario, que sean varias (portavocía coral). En segundo lugar, la otra gran pregunta que hay que hacerse a la hora de designar a nuestro portavoz de crisis es si tiene que responder a un perfil de tipo técnico o de tipo político, primando bien la función de liderazgo político o la del expertise profesional.

Este doble debate que acabamos de plantear supone uno de los hilos conductores del presente apartado, aplicado a un contexto concreto, la gestión de la crisis de la Covid-19 por parte del Gobierno de España.

\subsection{1. ¿Cuántos portavoces?}

En las decisiones adoptadas por el Gobierno de España se pueden identificar, además de a Fernando Simón (el director del Centro de Coordinación de Emergencias y Alertas sanitarias), imagen principal de la comunicación de esta crisis, al presidente del Gobierno, Pedro Sánchez y al ministro de Sanidad, Salvador Illa, hasta cuatro portavoces habituales más: Miguel Villarroya (Jefe del Estado Mayor de la Defensa), José Ángel González (Comisario General de la Policía Nacional), Laurentino Ceña (Teniente General de la Guardia Civil) y María José Rallo (Secretaria General de Transportes y Movilidad). Si en la primera fase de la crisis (enero-febrero de 2020), las comparecencias eran protagonizadas en exclusiva por Fernando Simón, reforzando la comunicación de Simón el ministro Illa con entrevistas en medios, tras la declaración del estado de alarma, a Simón comenzaron a acompañarle los miembros del llamado 
La figura del portavoz en la comunicación de una crisis sanitaria: el caso del gobierno español durante la COVID-19

"Comité de Gestión Técnica", responsables políticos de segundo nivel o funcionarios con altos cargos pertenecientes a diversas áreas temáticas relevantes. A los miembros del Comité de Gestión Técnica se sumaron comparecencias recurrentes de los diferentes ministros (hasta 53 entre el 15 de marzo y el 26 de abril), especialmente de aquellos miembros del Gobierno que conformaron el "Mando único", y dos ruedas de prensa de los "responsables sanitarios": María José Sierra, del Centro de Coordinación de Alertas y Emergencias Sanitarias (Sierra ha llegado a sustituir a Simón como portavoz principal cuando este enfermó); Patricia Lacruz (directora general de Cartera Básica y Farmacia del Ministerio de Sanidad); María Jesús Lamas (directora de la Agencia Española del Medicamento); Raquel Yotti (directora del Instituto de Salud Carlos III) y Rodrigo Gutiérrez (director general de Ordenación Profesional del Ministerio de Sanidad).

En total, han ejercido como portavoces en algún momento de la crisis nueve personas, más el presidente del Gobierno y los ministros. No todos los portavoces han tenido la misma relevancia ni sus apariciones han sido igual de recurrentes. Algunos solo han aparecido en un par de ocasiones y ha sido, sin duda, Fernando Simón el portavoz principal de lo que podría ser identificado como un sistema de portavocía coordinada, múltiple y mixta. La parte técnica la ha liderado el doctor Simón y la parte política ha recaído en el propio presidente y en el ministro de Sanidad.

Aunque lo recomendable es que el portavoz sea único (Cf. Costa-Sánchez y LópezGarcía, 2020: 5) para evitar duplicidades y un discurso caótico, la estrategia gubernamental ha optado por presentar a la sociedad múltiples voces, si bien no todas ellas estaban en un mismo plano jerárquico. Es importante que los distintos actores que gestionan (y sufren) la crisis sepan quién es el portavoz oficial del gobierno, "alguien con prestigio y reconocimiento en la sociedad, no cualquier político improvisado" (Cf. Medina, 2017, p. 64). De lo que se trata es de que los receptores de la comunicación sepan "a quién deben dirigirse para demandar información" (Cf. Medina, 2017, p. 64). En este sentido, incluso con la presencia de un amplio reparto de portavoces puntuales, el Gobierno de España habría acertado en la designación de Fernando Simón como portavoz principal pues así ha sido reconocido por el conjunto de la ciudadanía, llegando a convertirse en foco tanto de los elogios (en un proceso de generación de branding personal digno de análisis) como de las críticas de la sociedad y los grupos políticos.

El modelo de portavocía coral ha generado momentos de confusión, como derivaciones continuas de una misma pregunta entre distintos portavoces, fruto del desconocimiento de estos de las áreas competenciales de los demás. Si el objetivo de la comunicación de crisis es dotar de certidumbre a las personas que te escuchan, ver a los ministros pasándose preguntas, desde luego, no es la mejor estrategia. Por probabilidad, es más fácil que haya errores cuantos más portavoces comparezcan tanto a la vez como a lo largo del tiempo. Con todo, aun contraviniendo la teoría, es comprensible que el Ejecutivo español optara por tener varios portavoces dado el carácter tan transversal de esta crisis, que afectaba a muchas labores gubernamentales, no por la cuestión sanitaria, sino por las consecuencias derivadas de ella. 
Todo lo anterior se concluye partiendo de la base de que la comunicación (también la de crisis y la de riesgo) ha de estar adaptada a su contexto y este contexto ha sido una verdadera "crisis total" que ha puesto a prueba las teorías más asentadas académicamente. En general, es recomendable que el número de portavoces sea el menor posible y, si es único, mejor. Primero, porque es más sencillo homogeneizar y preparar el mensaje cuando los portavoces son pocos; segundo porque, para el público, que coexistan dos o más voces emisoras, enrarece la comunicación como cuestión general; tercero, porque cada persona comunica de una forma y es importante que los receptores del mensaje (los ciudadanos) se adapten a la forma de comunicar de un portavoz para que cada vez tengan más facilidad a la hora de comprender el mensaje, y, cuarto, porque ocurre lo mismo con los medios de comunicación: no todos los políticos, portavoces y expertos se relacionan igual con los medios y es fundamental que haya cierta sinergia para que la "traducción" de nuestro mensaje sea lo más fiel posible.

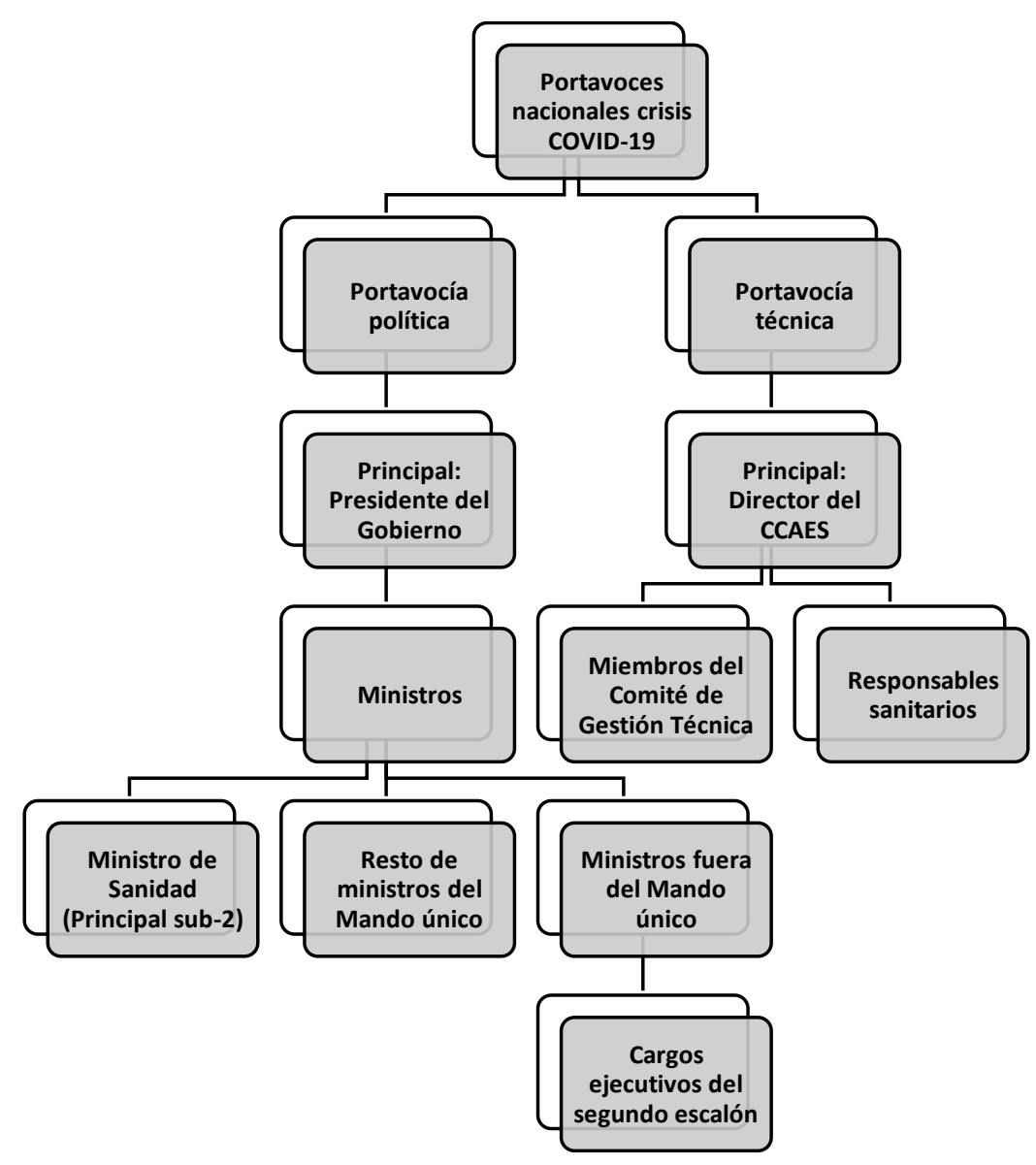

Figura 1. Representación esquemática del sistema de portavocía múltiple coordinada mixta en la crisis sanitaria por la COVID-19 a nivel del Gobierno de España.

Fuente: elaboración propia. 
La figura del portavoz en la comunicación de una crisis sanitaria: el caso del gobierno español durante la COVID-19

\subsubsection{El perfil del portavoz}

Hablemos primero de la elección del portavoz técnico. La persona que representa a la perfección esta figura no es otra que Fernando Simón. Uno de los fundamentos más relevantes para que el portavoz de la crisis sea un experto o un técnico es que aporta legitimidad al proceso de gestión. Como es evidente, que quien hable a los ciudadanos sea un profesional de la materia genera tranquilidad y certidumbre en la población, aporta confianza y la confianza es un bien muy valioso para cualquier organización en tiempos de crisis.

El principal problema del portavoz técnico aparece de su propia virtud: expone el criterio técnico (Aboud, 2020). Si el portavoz es una de las personas que más sabe sobre la materia que centra la crisis, lo lógico es que tenga algún tipo de poder decisorio. Al comparecer públicamente se le expone y se facilita que los medios de comunicación y la opinión pública en general cuestionen más las decisiones y las medidas adoptadas. Se le pone cara al experto principal y eso puede llegar a condicionar la buena gestión por una suerte de síndrome de burnout mediático. El portavoz técnico es el que aporta el conocimiento necesario para salir (y salir sin daños) de la crisis (Aboud, 2020). Es, por tanto, un valor demasiado importante como para exponerlo a la crítica pública.

Existe una cuestión para la que el portavoz técnico sí podría ser la mejor opción: la comunicación de riesgo. Al ser el mayor conocedor de la materia, de los protocolos y de los riesgos reales, es el que mejor puede trasladar a la población las recomendaciones, las conductas pautadas y la concienciación sobre los peligros. No podemos olvidar que el desgaste generado por una crisis es exponencialmente mayor al propio de la gestión ordinaria, ya que los efectos de la crisis impactan de una manera tan agresiva en la población que se disparan las emociones. Para un funcionario o experto, sin embargo, este es un peligro mucho menor, ya que al no ser elegido por la población y no presentarse a unas futuras elecciones su posición no depende de su buena valoración pública. Eso sí, los efectos a nivel personal deben ser muy tenidos en cuenta.

A veces sucede que un portavoz técnico, un científico, puede resultar un fenómeno comunicativo. Fernando Simón ha sido la figura del portavoz técnico en esta crisis y también el portavoz más importante de la misma. Simón llega como una persona en principio desideologizada y apartidista. Había sido portavoz en la crisis del ébola, gestionada por un gobierno de otro color político y esto, además, le hacía algo conocido en todo el país. Sin embargo, la evolución de la portavocía de Simón ha sido ciertamente novedosa respecto a otras crisis analizadas por la literatura: se ha convertido en todo un fenómeno en redes sociales, lo que algunos llaman ya un icono pop (Gil, 2020). Simón no es ni se espera de él que sea un especialista de la comunicación política, y es algo que ha jugado en favor de su imagen pública. Su naturalidad y espontaneidad han sido un valor clave que ha provocado que en redes como Twitter los usuarios hayan creado miles de memes (normalmente en positivo) con la figura del portavoz, como señalaba Gil (2020). Al final, el técnico ha logrado transmitir una naturalidad y un liderazgo que serían propios del ámbito político. No obstante,

Revista de Comunicación y Salud, 2020, Vol. 10, nº 2, pp. 397-416 
aunque el "fenómeno pop" de Simón nos pueda resultar simpático y agradable, corresponde a un fenómeno de politización conflictiva que genera el riesgo de debilitar su criterio técnico.

Pasando a la elección del portavoz político, es importante recalcar que los objetivos de su actuación son esencialmente diferentes a los del técnico. El portavoz político de esta crisis, sin contar con la figura del presidente, ha sido el ministro de Sanidad, Salvador Illa. Compareció prácticamente a diario y fue el apoyo político del doctor Simón. Si bien lo clásico sería denominar como portavoz político exclusivamente al ministro Illa, lo cierto es que el presidente Pedro Sánchez ha ejercido de manera muy evidente de portavoz con unas extensas ruedas de prensa nunca antes vistas. $Y$ es lógico que haya sido así; la máxima figura del Ejecutivo debía comparecer para explicar las medidas de más alto rango como, por ejemplo, la declaración del estado de alarma (rueda de prensa del 13 de marzo). Lo que podemos concluir es que, bien por las particularidades de la crisis, bien por la estrategia de Moncloa, la portavocía política ha sido también múltiple y jerárquica. El principal emisor de mensajes ha sido el presidente del Gobierno, posteriormente el ministro de Sanidad, luego los ministros del Mando Único (Interior, Defensa y Transportes) y, finalmente, el resto de miembros del Ejecutivo.

Por último, la selección de un portavoz político puede ser interesante teniendo en cuenta la idea de la crisis como una oportunidad para el liderazgo, en tanto a momento de exposición y atención pública intensivo (2003, p. 140). Muchos políticos, afirma Canel (2010, p. 244), han mejorado y reforzado su liderazgo ante una situación tremendamente complicada que finalmente gestionaron con éxito. Tal vez el caso más reciente sea el del alcalde de Madrid, José Luis Martínez Almeida, portavoz de crisis de su institución y líder que ha sabido rentabilizar la gestión de un escenario a priori negativo.

Tabla 2. Los distintos perfiles del portavoz.

\begin{tabular}{c|l|l}
\hline Tipo de portavoz & \multicolumn{1}{|c|}{ Atributos } & \multicolumn{1}{|c}{ Ejemplos } \\
\hline Portavoz político & $\begin{array}{l}\text { Liderazgo, } \\
\text { dimensión } \\
\text { ideológica, } \\
\text { autoridad. Más } \\
\text { capacidad } \\
\text { comunicativa. }\end{array}$ & $\begin{array}{l}\text { Alfredo Pérez } \\
\text { Rubalcaba, } \\
\text { vicepresidente del } \\
\text { Gobierno en la crisis } \\
\text { de los controladores } \\
\text { aéreos (2010). }\end{array}$ \\
\hline Portavoz técnico & $\begin{array}{l}\text { Legitimidad } \\
\text { racional, } \\
\text { objetividad, base } \\
\text { científica, } \\
\text { confianza. }\end{array}$ & $\begin{array}{l}\text { Fernando Simón, } \\
\text { de la crisis del ébola } \\
(2014) \text { y en la crisis } \\
\text { de la COVID-19 } \\
(2020)\end{array}$ \\
& & \\
\hline
\end{tabular}

Fuente: elaboración propia. 
La figura del portavoz en la comunicación de una crisis sanitaria: el caso del gobierno español durante la COVID-19

La dicotomía técnico-político abarca, igualmente, ciertas cuestiones relativas a las dotes comunicativas de cada uno, pero estas serán tratadas en otro apartado 3.3 del presente trabajo.

\subsection{La relación entre el portavoz y los medios}

Como en todo proceso político, la comunicación de una crisis ha de tener en cuenta el rol jugado por los medios. No son únicamente transmisores de información; políticamente, sus acciones pueden reforzar o debilitar la posición del Gobierno y, desde un punto de vista pedagógico, la acción necesaria de concienciación social ante la crisis requerirá de su colaboración activa.

¿Cómo debemos, entonces, entender la relación entre los medios y el portavoz? En línea con la teoría revisada, toda comunicación que realice el Gobierno en estos contextos debe orientarse a la generación de certidumbres. Ello implica asumir que una situación de crisis genera dudas en la ciudadanía, de las cuales los medios serán los portavoces, hecho que obliga a que la relación con los mismos no sea unidireccional, sino dialógica (Cf. Costa-Sánchez y López-García, 2020: 5), respondiendo sistemáticamente a sus preguntas. Esta escucha y respuesta por parte del Gobierno se debe guiar por una transparencia escrupulosa, que no dé pie a la sensación de que el ejecutivo trata de evitar la rendición de cuentas.

La disponibilidad y resolución de dudas por parte del Gobierno hacia los medios debe tener cierta profundidad y ser intensiva. El establecimiento de procesos o filtros complejos para poder acceder al portavoz de la crisis es de todo punto contraproducente. A los medios, por tanto, se les debe facilitar su labor de control y consulta al Ejecutivo, no ya solo evitando los filtros mencionados sino adaptándose dentro de lo razonable a algunas de sus dinámicas (horarios de especial relevancia informativa, formatos favorecedores de las ruedas de prensa, etc.) y aportando seguridad en el trato con los mismos.

Nuestro análisis en este apartado partirá de los supuestos establecidos en los anteriores párrafos para mostrar una serie de indicadores que nos permitan concretar la idoneidad de la relación entre portavocía gubernamental y medios durante la crisis del coronavirus en España. La existencia de bidireccionalidad entre portavocía y medios se medirá contabilizando las comparecencias en las que se aceptaron preguntas sobre el total de comparecencias; la intensidad de la dinámica de preguntas y respuestas, a través de indicadores como el número medio de preguntas aceptadas por comparecencia, la ratio entre tiempo total de comparecencia y duración del turno de preguntas o las entrevistas concedidas a medios. Consideraremos finalmente la adaptación del Gobierno a las pautas de los medios y la facilitación de su labor fijándonos en el cumplimiento de la hora anunciada de comparecencia y en el formato establecido para las ruedas de prensa. Los indicadores se aplicarán, por un lado, a las comparecencias del comité técnico y, por otro, a las del propio presidente del Gobierno. Como fuente hemos empleado el informe Comparecencias sobre el coronavirus COVID 19 tras la declaración del estado de alarma elaborado por la Secretaría de Estado de

Revista de Comunicación y Salud, 2020, Vol. 10, ํㅡ 2, pp. 397-416 
La figura del portavoz en la comunicación de una crisis sanitaria: el caso del gobierno español durante la COVID-19

Comunicación, y los vídeos de las ruedas de prensa subidos a la plataforma YouTube por diversos medios de comunicación.

En el caso del presidente del Gobierno (ver tabla 3), entre el 12 de marzo y el 26 de abril, se han contabilizado once comparecencias en un total de cuarenta y cinco días, de las cuales diez tuvieron turno de preguntas, con una duración media aproximada de una hora y cerca de la mitad del tiempo dedicado a responder a las cuestiones planteadas por los periodistas, rondando los nueve turnos por comparecencia. Es innegable que ha habido reciprocidad en la comunicación entre Pedro Sánchez, en su función de portavoz político de la crisis, y los medios de comunicación, así como una cierta profundidad en la misma. La cara negativa de estas cifras es cómo la abundancia de comparecencias presidenciales (una cada cuatro días de media) sumada a su extensa duración puede provocar un efecto de saturación informativa, que haga al ciudadano perder el interés o aumentar su valoración negativa de la gestión.

Tabla 3. Indicadores de la relación entre portavoces y medios para Pedro Sánchez ${ }^{2}$

\begin{tabular}{ll}
\hline Número de comparecencias & 11 \\
\hline Comparecencias con turno de pregunta & $10 / 11$ \\
\hline Duración media de las comparecencias & $00: 54: 28$ \\
\hline Duración media turno preguntas & $00: 29: 19$ \\
\hline$\%$ medio de tiempo de comparecencia dedicado a preguntas & $53,78 \%$ \\
\hline Media de turnos de pregunta por comparecencia & 8,7 \\
\hline
\end{tabular}

Fuente: elaboración propia.

Por otra parte, no incluimos en la tabla las cuestiones del formato del turno de preguntas en las comparecencias o el cumplimiento de la hora anunciada de comparecencia por su difícil cuantificación, pero son igualmente indicadores de nuestro estudio. Cabe destacar, primeramente, los múltiples retrasos de las comparecencias presidenciales ante la prensa (en algunos casos llegaron a ser de horas). Pero lo más notorio tal vez haya sido el método de selección de preguntas en las ruedas de prensa. Criticado por diversos medios y periodistas ${ }^{3}$, inicialmente este consistía en la remisión vía WhatsApp de las preguntas a un chat abierto por la Secretaría de Estado de Comunicación, cuyo responsable, Miguel Ángel Oliver, escogía aquellas que considerara "representativas de los temas más reiterados". Posteriormente, estas cuestiones eran formuladas en voz alta por el propio Secretario de Estado en las ruedas

${ }^{2}$ Excluimos del cálculo del porcentaje de tiempo de la comparecencia dedicado a preguntas y de la duración media de la comparecencia la aparición televisiva de Pedro Sánchez el 13 de marzo, pues en esta no se admitieron preguntas y tuvo un carácter atípico, apenas siete minutos dedicados en exclusiva a comunicar la declaración del estado de alarma.

3 Palomera, E., \& Castro, I. (2020). Ruedas de prensa en tiempos de pandemia: Moncloa selecciona preguntas por escrito y Arrimadas y Casado responden por videollamada. Eldiario.es. Recuperado de https://www.eldiario.es/politica/coronavirus-moncloa-videollamadas-casado-arrimadas 1 1009263.html

Revista de Comunicación y Salud, 2020, Vol. 10, nº 2, pp. 397-416 
La figura del portavoz en la comunicación de una crisis sanitaria: el caso del gobierno español durante la COVID-19

de prensa presidenciales para su respuesta por parte de Pedro Sánchez ${ }^{4}$ o del portavoz correspondiente. El conocimiento previo de las preguntas permitía a los miembros del gobierno planificar respuestas e, implícitamente, establecía un filtro arbitrario mediante el cual el Gobierno podía seleccionar solo las preguntas más convenientes. Este sistema contrastaba con los escogidos por otros líderes políticos de la oposición y por los gobiernos de otros países. Tras la polémica generada, el Gobierno modificaría el proceso de selección de preguntas ${ }^{5}$, permitiendo a los periodistas entrar en directo mediante una videollamada y poder formular así sus preguntas a los miembros del Gobierno, repartiendo los turnos de palabra de forma aleatoria entre los medios habituales.

Para el análisis de las comparecencias del Comité Técnico, las fuentes empleadas son las mismas. La primera comparecencia analizada se celebró en el 16 de marzo y la última el 26 de abril.

Al tratar cuestiones más relativas a la gestión cotidiana de la pandemia (asuntos menos trascendentales y solemnes), el Comité Técnico comparece con más frecuencia que el presidente del Gobierno. Salvando un día del período analizado, la dinámica que siguió fue la comparecencia diaria, con cuarenta y una ruedas de prensa en total durante el periodo analizado. La duración media ronda los tres cuartos de hora, algo menor a las comparecencias presidenciales y, tanto la extensión media del turno de preguntas, como el porcentaje del mismo sobre el total de la comparecencia es menor que en el caso de las apariciones del presidente. Eso sí, la media de preguntas respondidas es prácticamente la misma.

Tabla 4. Indicadores de la relación entre portavoces y medios para el Comité Técnico.

\begin{tabular}{ll}
\hline Número de comparecencias & 41 \\
\hline Comparecencias con turno de pregunta & $41 / 41$ \\
\hline Duración media de las comparecencias & $00: 47: 05$ \\
\hline Duración media turno preguntas & $00: 18: 21$ \\
\hline$\%$ medio de tiempo de comparecencia dedicado a preguntas & $38,70 \%$ \\
\hline Media de turnos de pregunta por comparecencia & 8,87 \\
\hline
\end{tabular}

Fuente: elaboración propia.

De nuevo observamos que (tabla 4), efectivamente, se da la citada bidireccionalidad entre portavoces técnicos y prensa, con un nivel apreciable de profundidad (ruedas de prensa extensas y diarias). Los escollos en la relación entre portavocía y medios se darían, de nuevo, en el formato de preguntas. Aun cuando en el caso del Comité

\footnotetext{
${ }^{4}$ Los periodistas no se encontraban presencialmente en la sala, dada la obligación de permanecer en casa y evitar aglomeraciones.

5 El Gobierno rectifica y permitirá preguntas directas de los periodistas. (2020). El País. Recuperado de https://elpais.com/espana/2020-04-05/el-gobierno-rectifica-y-permitira-preguntas-directas-de-losperiodistas.html.
}

Revista de Comunicación y Salud, 2020, Vol. 10, nº 2, pp. 397-416 
La figura del portavoz en la comunicación de una crisis sanitaria: el caso del gobierno español durante la COVID-19

Técnico no se generó la polémica que se produjo con las ruedas de prensa del jefe de Gobierno, al ser de carácter menos político, se repite el formato inicial de preguntas leídas por el Secretario de Estado de Comunicación. En el caso del Comité, también ha existido, en ocasiones, retrasos en la hora anunciada de comparecencia.

Antes de concluir este apartado, es importante señalar que, para una correcta relación con los medios, es recomendable que los portavoces realicen una formación en media training, pues aporta habilidades comunicativas a la hora de dirigirse a los medios de comunicación y ciudadanos que son fundamentales en la formación de un portavoz.

La construcción del mensaje aparece como uno de los factores más importantes que deben ser entrenados (Cf. Sánchez, 2016, p. 183). Pueden usarse, como si de la comunicación electoral se tratara, argumentarios o recopilaciones de réplicas y se deben cuidar algunos aspectos como la claridad del lenguaje, la no divagación o la mesura a la hora de aportar cifras (Cf. Sánchez, 2004, p. 95).

Para el éxito de los mensajes, también es importante elegir correctamente el canal que se usa en su emisión, primando en situaciones de crisis "las vías unipersonales más directas y rápidas", acudiendo a "modelos múltiples (rueda) o individuales (mensajes a cada uno de los medios, incluidas las agencias)" (Moreno, 2008, p. 121). Por la necesidad de informar en tiempo real y con un cierto grado de inmediatez, es preciso hacer las apariciones en directo. Estudiando el desempeño en este terreno del Gobierno de España ante la crisis de la Covid-19, encontramos que el principal modelo elegido para trasladar los mensajes ha sido la rueda de prensa telemática en directo, para todos los medios, emitida también en streaming por redes, todo ello desde el Palacio de la Moncloa. También ha sido destacable la planificación de entrevistas en medios de los diversos portavoces políticos de la crisis.

Con todo, durante esta pandemia, el Gobierno ha hecho uso de otras formas de comunicación que solapaban la comunicación de crisis y la de riesgo con la comunicación gubernamental. El Ejecutivo ha realizado una importante campaña de comunicación institucional con spots, publicidad y lemas ${ }^{6}$ que mezclaban la concienciación contra el virus (comunicación de riesgo) con el relato político que se quería instalar en la sociedad.

\subsection{Dotes comunicativas y poder decisorio en la portavocía}

Dentro de las múltiples alternativas a la hora de concretar la portavocía de la crisis, una de las cuestiones que se plantean es cuándo el portavoz debería tener un perfil marcadamente comunicativo o bien ser una de las personas con autoridad a la hora de decidir en la gestión de la crisis. Esta dicotomía profundiza la que clásicamente se viene

${ }^{6}$ Con la utilización de un relato y eslóganes al modo de la comunicación gubernamental, el Gobierno de España ha desafiado la ausencia eslóganes que, según la teoría (Riorda, 2011, p. 38), caracteriza a la comunicación de crisis.

Revista de Comunicación y Salud, 2020, Vol. 10, nº 2, pp. 397-416 
La figura del portavoz en la comunicación de una crisis sanitaria: el caso del gobierno español durante la COVID-19

discutiendo sobre la portavocía técnica o política y que hemos tenido ocasión de abordar en el apartado 3.1.2.

En el primer caso, estaríamos hablando de profesionales de la comunicación o del periodismo, personal con formación o experiencia específica en el ámbito mediático. Las ventajas son evidentes; el portavoz sabrá comunicar con naturalidad y evitar errores ante los medios causados por la falta de experiencia con los mismos. No obstante, está claro que alguien del perfil que indicamos no tiene por qué ser un experto conocedor de la materia sobre la que versa la crisis, además de poder dar la sensación de que, escogiendo una persona encargada exclusivamente de la función de portavoz, el Gobierno evita dar la cara personalmente.

Respecto a la elección de una persona con autoridad decisoria en la gestión de crisis, debemos considerar que puede ser lo esperable por parte de la ciudadanía (aquel que toma las decisiones rinde cuentas de las mismas), pudiendo generar además cierta sensación de seguridad por su posición en la jerarquía. No obstante, un cargo de este tipo normalmente será político y, en contextos de democracias con altas tasas de desafección, esto pondrá en duda la credibilidad del portavoz. Así, para ciertas situaciones complicadas, la ciudadanía puede preferir a personas menos vinculadas políticamente, lo cual, además, evita el riesgo de que el cargo político escogido "se queme". Debemos distinguir, además, la rendición de cuentas política de la portavocía de una crisis.

En el caso español, las comparecencias realizadas por ministros o el propio presidente corresponden al modelo de portavocía política del cargo con poder decisorio ("el que gestiona, explica y comparece"). En los momentos álgidos, la aparición de Pedro Sánchez era prácticamente un requerimiento obligatorio, dada la magnitud que había tomado la crisis. Más cuestionable es la aparición de distintos ministros más allá del de Sanidad pues, si bien estos trataban cuestiones relativas a sus departamentos, se generaba una multiplicación de la cantidad de portavoces poco eficiente a nivel comunicativo.

Respecto a las comparecencias de Fernando Simón y de otros miembros de Comité Técnico sin un cargo político en el Gobierno, debemos matizar la cuestión del poder decisorio. Todos los miembros del Comité Técnico ocupan un lugar importante en la jerarquía de la gestión de la crisis, pero el poder último de decisión corresponde a la escala política ministerial. Sin duda, la comunicación realizada por portavoces de este perfil alejaba (al menos en un principio) la desconfianza, pero llegaron a darse casos de polémicas provocadas por la poca habilidad de algunos de los portavoces en la relación con los medios (recordemos, por ejemplo, la alusión a la persecución de bulos contra el Gobierno por parte del representante de la Guardia Civil).

\section{CONCLUSIONES}

La pandemia global provocada por la COVID-19 puede entenderse como una "crisis total" que ha puesto a prueba todos los manuales de comunicación existentes. En este

Revista de Comunicación y Salud, 2020, Vol. 10, oㅡ 2, pp. 397-416 
La figura del portavoz en la comunicación de una crisis sanitaria: el caso del gobierno español durante la COVID-19

contexto novedoso, que no encuentra paralelismo con ninguna situación de amenaza sanitaria en los últimos años, la figura del portavoz ha sobresalido como elemento fundamental para la estrategia comunicativa de los gobiernos.

España ha sido uno de los países más afectados por el coronavirus y también donde se han adoptado decisiones más restrictivas por parte del Gobierno. Transmitir los mensajes correctos, informar sobre la evolución de la situación y trasladar recomendaciones y normas a la población resulta una labor tan complicada como imprescindible para controlar la expansión del virus y poder cerrar la crisis en el menor tiempo posible. El Ejecutivo español ha desafiado a la teoría sobre portavocía de crisis y ha optado por un modelo con múltiples portavoces y mixto, incluyendo al mismo tiempo y solapadamente tanto a personas de perfil político como a técnicos, en un ejercicio de especialización que conecta con el carácter transversal de la crisis. Sin embargo, el sistema coral de portavocía ha generado con demasiada frecuencia ruido informativo, saturación y contradicciones. Además, muchos de los portavoces no contaban con excesivas destrezas en materia de comunicación y medios. Todo ello nos lleva a reafirmar la necesidad de transformar y resignificar, para la función de portavocía, el concepto de experto desde la concepción actual de persona que profesionalmente domina la materia hasta un perfil de experto en comunicación que traslade con eficacia los mensajes construidos, sin perjuicio de que sean los científicos quienes conformen los comités de gestión y toma de decisiones. Los fallos detectados en la comunicación del Gobierno español a buen seguro podrían haberse minimizado con un sistema de portavocía más simple (tendiendo casi al portavoz único) y con primacía de la vertiente comunicativa sobre la técnica, exclusivamente en el ámbito mediático, no en el de gestión.

\section{REFERENCIAS}

Aboud, L. (27 de abril de 2020). Portavoces técnicos sí, pero sin abusar ni quemarlos. El Confidencial. Recuperado de: https://blogs.elconfidencial.com/espana/tribuna/2020-04-27/portavoces-tecnicos-sipero-sin-abusar-ni-quemarlos 2567091/.

Beck. U. (1992). Risck Society. Towards a New Modernity. Londres: Sage Publications.

Canel, M. J. (2010). Comunicación de las instituciones públicas. Madrid: Tecnos.

Consejo de Seguridad Nuclear -CSN- (2012). Manual de comunicación de crisis para las organizaciones reguladoras nucleares. Madrid: Consejo de Seguridad Nuclear.

Costa-Sánchez, C. y López-García, X. (2020). Comunicación y crisis del coronavirus en España. Primeras lecciones. El Profesional de la Información, 29 (3), 1-14, e290304. doi: 10.3145/epi.2020.may.04 
La figura del portavoz en la comunicación de una crisis sanitaria: el caso del gobierno español durante la COVID-19

Crespo, I., Garrido, A. y Medina, R.M. (2017). La comunicación de crisis en la Administración pública española: análisis de la evidencia empírica. GAPP, 18, 110 134. doi: $\underline{10.24965 / g a p p . v 0 i 18.10465}$

Crespo, I. y Garrido, A. (2020). La pandemia del coronavirus: estrategias de comunicación de crisis. Más Poder Local, 41, 12-19. Recuperado de http://maspoderlocal.es/files/articulos/coronavirus-estrategias-comunicacion-crisis$\underline{\mathrm{mpl}}$ 41.pdf

El País. (5 de abril de 2020). El Gobierno rectifica y permitirá preguntas directas de los periodistas. El País. Recuperado de https://elpais.com/espana/2020-04-05/elgobierno-rectifica-y-permitira-preguntas-directas-de-los-

periodistas.html?outputType=amp.

Fumanal, V. (2020). La portavocía: ¿Experta o política? En VV.AA., Comunicación política en tiempos de coronavirus (23-27). Barcelona: Cátedra Ideograma-UPF de Comunicación Política y Democracia.

Gil, F.G. (6 de mayo de 2020). Fernando Simón: un portavoz científico convertido en icono pop de Internet. Eldiario.es Recuperado de: https://www.eldiario.es/tecnologia/fernando-simon-portavoz-cientificofolclore 1 5967786.html.

Granda Revilla, J. (2015). Cómo aprender de los errores: comunicación en crisis sanitarias. MĖTODE Science Studies Journal, 88, 69-72. doi: 10.7203/metode.6.7509

Lijphart, A. (1971). Comparative Politics and the Comparative Method. APSR, 65, 682693. Recuperado de https://www.jstor.org/stable/1955513

Cerdá, J.C. (2011). El riesgo de una mala comunicación de riesgos. Revista de

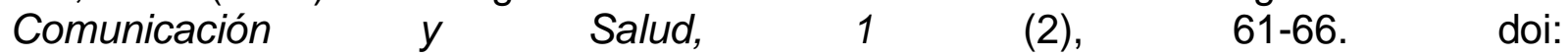
$\underline{10.35669 / \text { revistadecomunicacionysalud.2011.1(2).61-66 }}$

Medina Mínguez, R. M. (2017). Comunicación en situaciones de crisis: factores de éxito que favorecen su eficacia: estudio comparado de casos de comunicación de crisis en el sector público español entre los años 2010-2014 (Tesis doctoral Universidad de Murcia). Recuperado de la base de datos digitum.um.es: https://digitum.um.es/digitum/bitstream/10201/52892/1/TESIS\%20ROSA\%20MARIA \%20MEDINA\%202017.pdf.

Moncloa (26 de abril de 2020). Comparecencias sobre el coronavirus COVID-19 tras la declaración del estado de alarma. Recuperado de https://www.lamoncloa.gob.es/serviciosdeprensa/notasprensa/presidencia/Paginas/2 020/260420-comparecencias.aspxm. 
La figura del portavoz en la comunicación de una crisis sanitaria: el caso del gobierno español durante la COVID-19

Moreno, E. (2008). Gestión de la información y la comunicación en emergencias, desastres y crisis sanitarias. Emergencias. Revista de la Sociedad Española de Medicina de Urgencias y Emergencias, 20 (2), 117-124.

Olsson, E. K. (2014). Crisis Communication in Public Organisations: Dimensions of Crisis Communication Revisited. Journal of Contingencies and Crisis Management, 22 (2), 113-125. doi: $\underline{10.1111 / 1468-5973.12047}$

Organización Panamericana de Salud. La comunicación de riesgo: preguntas frecuentes. Pan American Health Organization / World Health Organization. Recuperado 12 de mayo de 2020, de https://www.paho.org/hq/index.php?option=com content\&view=article\&id=11400:lacomunicacion-de-riesgo-preguntas-frecuentes\&ltemid=41610\&lang=es

Palomera, E. y Castro, I. (24 de marzo de 2020). Ruedas de prensa en tiempos de pandemia. Moncloa selecciona preguntas por escrito y Arrimadas y Casado responden por videollamada. Eldiario.es. Recuperado de https://eldiario.es/política/coronavirus-moncloa-videollamadas-casadoarrimadas 1 1009263.html.

Paniagua Gallart, C. (2012). Una historia de la comunicación de crisis en España. Revista ICONO14 Revista Científica De Comunicación Y Tecnologías Emergentes, 8(2), 3-24. doi: https://doi.org/10.7195/ri14.v8i2.24

Peytibi, X. (2020). En momentos de incertidumbre, es la hora de los gobiernos. 10 necesidades en comunicación de crisis. En VV.AA., Comunicación política en tiempos de coronavirus (10-17). Barcelona: Cátedra Ideograma-UPF de Comunicación Política y Democracia.

Riorda, M. (2011). Gestionando certidumbres: la comunicación de crisis no es comunicación gubernamental, electoral ni de riesgo. En Elizalde, L., Fernández, D. y Riorda, M. (comps.), La gestión del disenso. La comunicación gubernamental en problemas (7-44). Buenos Aires: La Crujía.

Riorda, M. (2012). Quiero salir...sólo y si se puede, bien. Más Poder Local, 10, 38-40.

Riorda, M. (2020). Antes de comunicar el riesgo o la crisis: hay que distinguirlo. Más Poder Local, 41, 20-23.

Rojas Orduña, O. I. (2003). La comunicación en momentos de crisis. Comunicar: Revista Científica de Comunicación y Educación, 11(21), 137-140.

Rosas-Rodríguez, M.E., y Barrios-Puga, A. (2017). Comunicación de riesgo, cambio climático y crisis ambientales. Chasqui. Revista Latinoamericana de Comunicación, 136, 179-194. https://doi.org/10.16921/chasqui.v0i136.32 
La figura del portavoz en la comunicación de una crisis sanitaria: el caso del gobierno español durante la COVID-19

Saló Lloveras, N. (2008). La comunicación de crisis en la sociedad del conocimiento: el papel del portavoz. Revista de Comunicación, 7, 22-26.

Sánchez Calero, M. L. (2004). Comité de crisis: actores, roles y trabajo. ZER, Revista de Estudios de Comunicación, 9 (17), 85-99.

Sánchez Galicia, J. (2016) Entrenamiento en medios (media training). En Crespo, I., D’Adamo, O. y Mora, A., Diccionario enciclopédico de Comunicación política (182184). Madrid: Centro de Estudios Políticos y Constitucionales.

\section{AUTORES}

José Miguel Rojo Martínez

Graduado en Ciencia Política y Gestión Pública por la Universidad de Murcia con Premio Extraordinario. Máster en Análisis Político Aplicado especialidad "Comunicación política". Investigador en la Universidad de Murcia. Ha sido Becario de Colaboración en el Departamento de Ciencia Política.

Orcid ID: https://orcid.org/0000-0001-9491-4839

Google Scholar: https://scholar.google.es/citations?user=3sMJEYcAAAAJ\&hl=es

\section{Salvador Moreno Moreno}

Graduado en Ciencia Política y Gestión Pública por la Universidad de Murcia. Máster en Análisis Político Aplicado especialidad "Comunicación política". Analista político para distintos medios regionales y plataformas de divulgación sobre Ciencia Política. Formador del Aula de Debate de la Universidad de Murcia. Investigador en la Universidad de Murcia.

Orcid ID: https://orcid.org/0000-0003-3064-8257

Google Scholar: https://scholar.google.com/citations?user=Sk5-nrEAAAAJ\&hl=es

\section{Alejandro Soler Contreras}

Graduado en Ciencia Política y Gestión Pública por la Universidad de Murcia. Máster en Análisis Político Aplicado especialidad "Comunicación política". Investigador en la Universidad de Murcia.

Orcid ID: https://orcid.org/0000-0002-2298-1992

Google Scholar: https://scholar.google.com/citations?user=3SgyVcMAAAAJ\&hl=es 